\title{
Upper extremity monoparesis in an adololescent without obvious profound etiology
}

\begin{abstract}
Background: Spinal cord injury in children is associated with severe morbidity and immense socioeconomic burden. In spinal cord injury without radiologic abnormalities (SCIWORA), magnetic resonance imaging (MRI) can detect intramedullary or extramedullary pathologies or show absence of neuroimaging abnormalities. Pathogenesis of SCIWORA is based on the concept that the inherently elastic paediatric spinal column can afford significant degree of segmental dislocation, resulting in damage of the spinal cord, without any obvious spinal fracture or ligamentous disruption

Case presentation: The medical history of the patient includes only a delayed presentation of unilateral upper extremity weakness to a child after violent traction of neck and upper extremity. A Guilford-type brace was applied to the patient for 3 months and the neurological outcome was eventually relatively uneventful. The clinical and radiologic work-up, combined with the neurological findings, established the diagnosis of SCIWORA, and we are highlighting the clinical and radiological features.

Conclusion: Review of the literature, regarding SCIWORA cases, reveals that the recognition of only edema of the spinal cord, without evidence of haemorrhage, as a positive predictive factor. SCIWORA should be included in the differential diagnosis of cases with paresis of the extremities, even though the possible traumatic event may manifest a significant time delay from the clinical sequelae, as it was the case for our patient.
\end{abstract}

Keywords: SCIWORA, demyelination, monoparesis, delayed presentation, magnetic resonance images
Volume 5 Issue 6 - 2018

\author{
Dimitrios Panagopoulos \\ Department of Neurosurgery, University of Thrace-University \\ Hospital of Alexandroupoli, Greece
}

\begin{abstract}
Correspondence: Dimitrios Panagopoulos, Department of Neurosurgery, University of Thrace-University Hospital of Alexandroupoli, Greece, Tel 0030-698I-3286 28, Emaildimpanayop@gmail.com
\end{abstract}

Received: March 06, 2018 | Published: November 19, 2018

\section{Introduction}

Spinal cord injury (SCI) in children is a challenging condition for the treating surgeon and paediatrician. ${ }^{1}$ It is considered to be rare, with an estimated incidence of 4.6 per million children per year. ${ }^{2}$ Spinal cord injuries without radiologic abnormality (SCIWORA) were first described by Pang \& Wilberger ${ }^{3,4}$ in 1982 . Although now known to also occur in adults, they defined it as 'children with objective signs of myelopathy as a result of trauma, whose plain films of the spine, tomography, and occasionally myelography carried out at the time of admission showed no evidence of skeletal injury or subluxation.' Mechanisms of injury for paediatric SCIWORA usually are a form of blunt trauma, including motor vehicle related, falling, sports related and child abuse. ${ }^{5}$ The immediate radiologic visualization of the spine by plain radiograph or computed tomography (CT) is an essential step in children with SCI. ${ }^{6-8}$ In cases of a clinic radiologic mismatch, that is, patients presenting with SCI without radiologic abnormalities (SCIWORA), the acquisition of early magnetic resonance images of the spine is recommended. ${ }^{7}$ Magnetic resonance imaging (MRI) allows the further subdivision of SCIWORA into cases with detectable intramedullary or extramedullary pathology and those without neuroimaging abnormalities (SCIWONA). ${ }^{8}$ The definition of SCIWORA in children is (1) traumatic SCI with acute neurologic deficit; (2) absence of fractures and/or dislocations at any spinal level as assessed by plain x-ray, CT or MRI; and (3) an immature skeleton or age less than 18 years.

\section{Materials and methods}

A girl 6years old of Greek ethnicity was admitted because of gradually deteriorating unilateral paresis of the right upper extremity, established 3days before. A mechanism of violent traction of the ipsilateral upper extremity and the neck was mentioned a week before the event. . It was attributed to a fall from a horintonal bar during an athletic activity session. Neurological examination revealed a decrease in muscular strength of the extremity $(2 / 5)$, reduction of relevant deep tendon reflexes without obvious sensory abnormalities. According to AIS (American Spinal Injury Association impairment scale), her initial assesement score was Grade B. The medical, family, and psychosocial history including genetic information of the patient revealed nothing. Relevant past interventions were not reported. MRI of the cervical spine, performed immediately after admission, revealed a longitudinal area within the spinal cord with abnormal signal intensity. This was localized between $\mathrm{C} 3$ and $\mathrm{C} 7$ myelotomes, from the right side of the midline, in the anatomical localization of gray matter substance, without gadolinium enhancement (Figures 1A-1C).This is probably attributed to injury of the spinal cord with resultant demyelination, resembling the radiological appearance of syringomyelia. It did not reveal any evidence of bony or ligamentous structure injury or intra parenchymal hematoma (Figure 2A) (Figure 2B). Electromyography and nerve conduction studies of the upper extremities recognized, besides the centrally located, within the frontal horns of the cervical cord, dysfunction, abnormalities in the 
conduction velocity at the level of the cervical roots. Based on the medical history and the neuroradiological findings, diagnosis of SCIWORA was established. Flexion and extension radiographs of the cervical spine didn't reveal any latent ligamentous instability and a Guilford-type brace was applied to the patient for 3months (Figure 3A-3C).

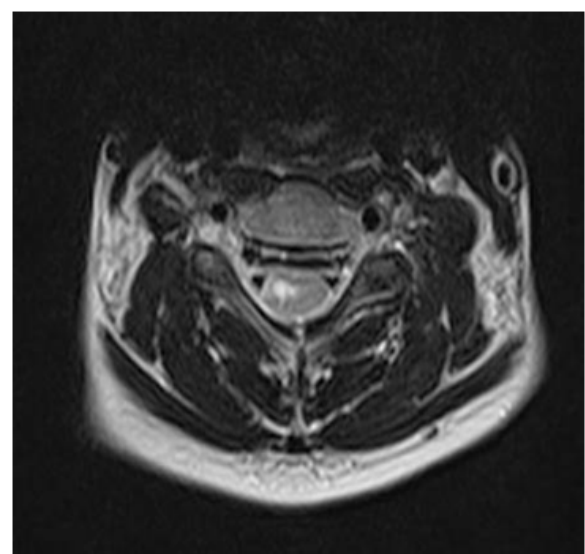

Figure I(A) Initial MRI scans (T2W, axial plane). It reveals a hyper intense signal in the spinal cord, in a longitudinal distribution, between C3and C7 myelotomes, suggestive of edema.

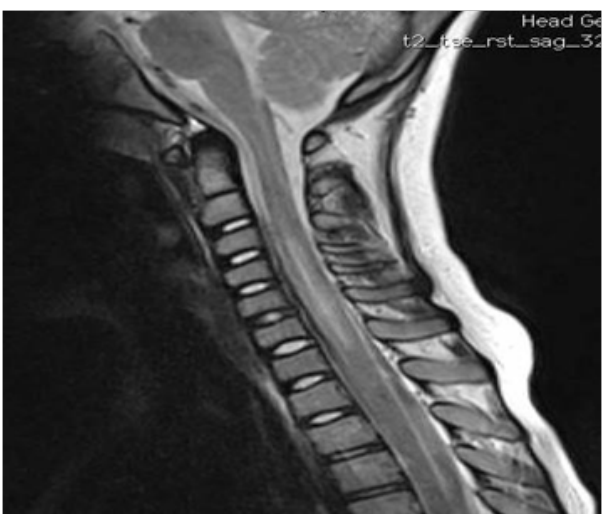

Figure I(B) Initial MRI scan (T2W, sagittal plane). It demonstrates the same findings, as Figure $\mathrm{I}(\mathrm{A})$.

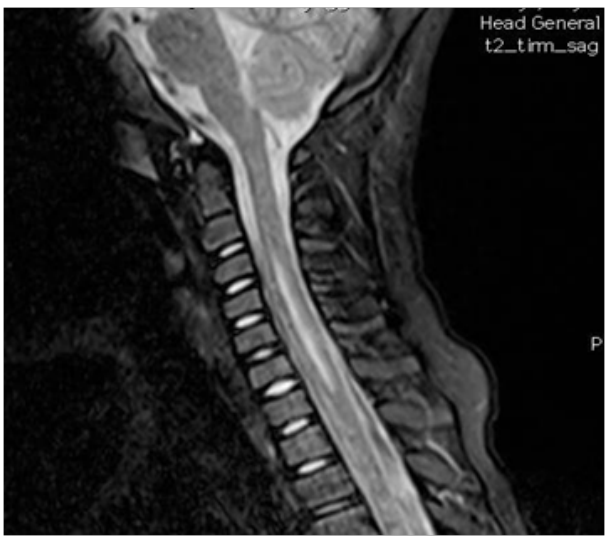

Figure I(C) Initial MRI scan (STIR, sagittal plane). It demonstrates the same findings, as Figure I(A).

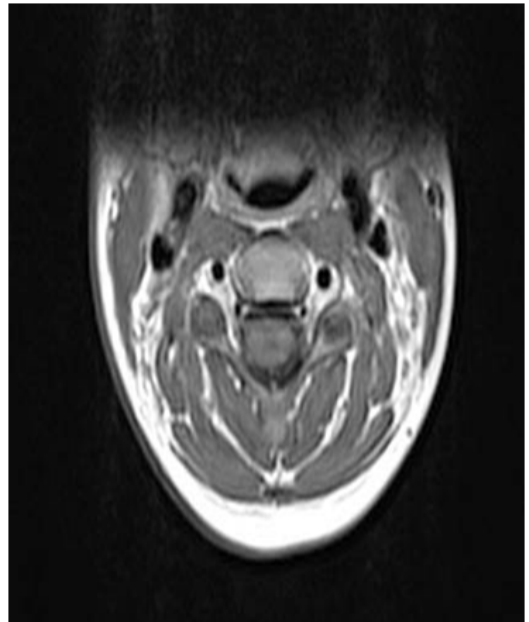

Figure 2(A) Initial MRI scan (TIW, axial plane). It depicts no evidence of hematoma, ligamentous disruption or vertebral fracture.

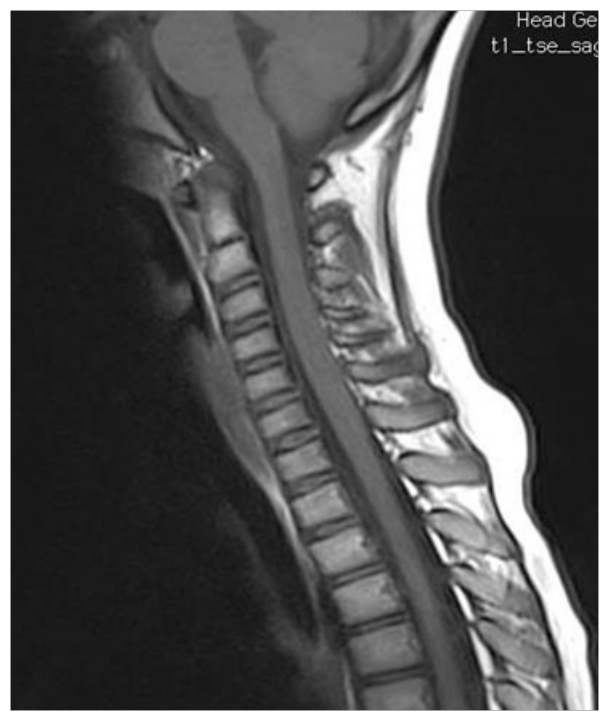

Figure 2(B) Initial MRI scan (TIW, sagittal plane). It depicts the same findings, as Figure 2(A).

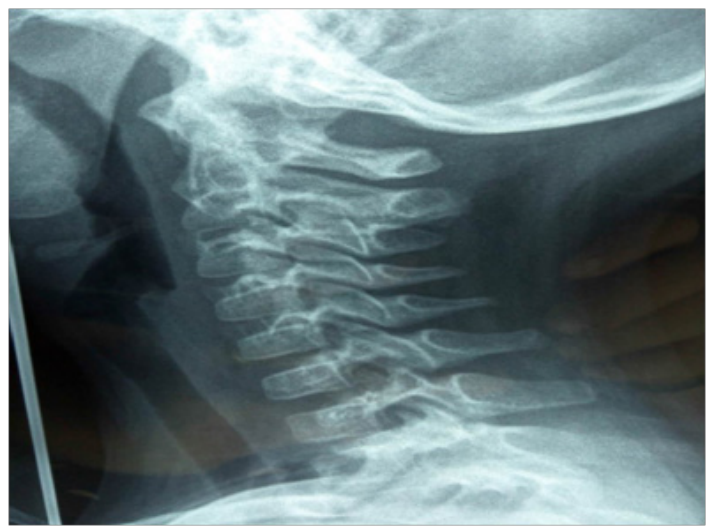

Figure 3(A) Initial lateral $\mathrm{X}$-ray of the cervical spine, in neutral position. It does not reveal any evidence of instability. 


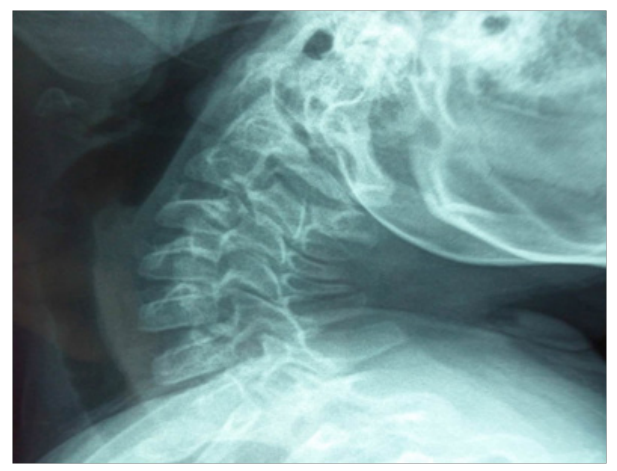

Figure 3(B) Initial lateral X-ray of the cervical spine, in extended position. This dynamic radiograph also does not reveal any evidence of instability.

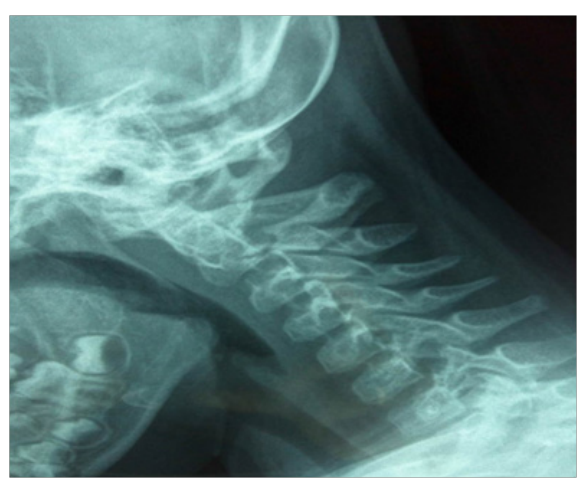

Figure 3(C) Initial lateral $X$-ray of the cervical spine, in flexed position (dynamic radiograph). It depicts the same findings, as Figures 3(A), Figure 3(B).

\section{Results}

Intervention adherence and tolerability of the patient was excellent, as we examined her at very close regular follow-up time periods. The patient was also re-examined clinically after that time period with clear signs of rehabilitation. Muscle strength was $5 / 5$ to all muscle groups, without abnormal deep tendon reflexes or superimposed sensory dysfunction. The total time period for full recovery to power was at 4 months after the insult. A repeat MRI scan was performed at that time point, which verified the disappearance of the abnormal signal changes, seen on previous imaging (Figure 4A) (Figure 4B).

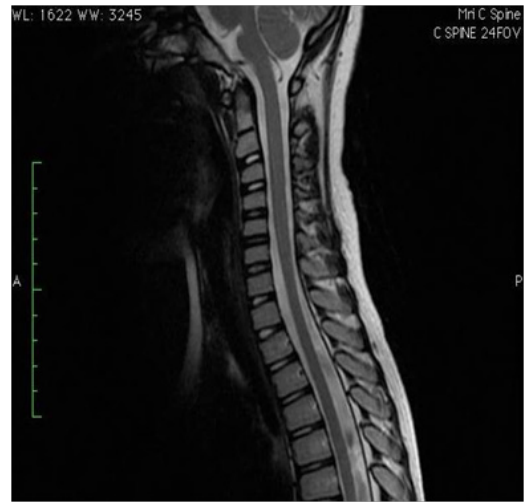

Figure 4(A) MRI scan 3 months after the insult, T2W, sagittal view. It depicts the disappearance of the signal changes indicative of spinal cord edema.

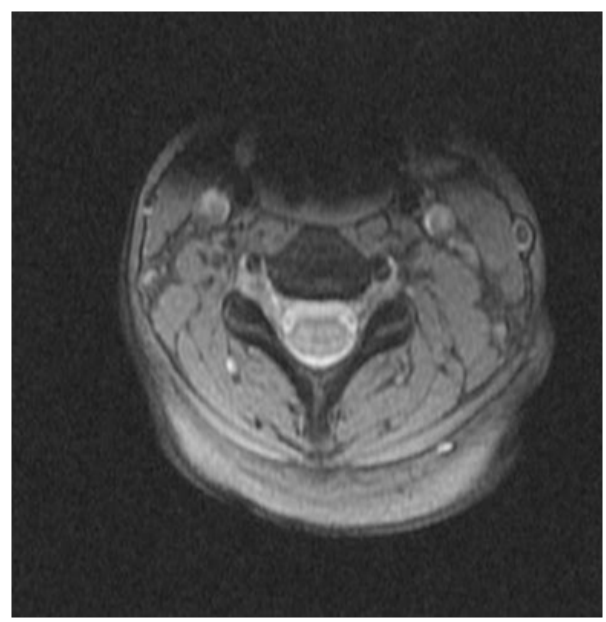

Figure 4(B) MRI scan 3 months after the insult, T2W, axial view. It depicts the same findings, as Figure 4(A).

\section{Discussion}

In traumatic SCI, it is critical to establish an early and reliable diagnosis to guide therapeutic interventions. Plain radiographs and CT remain the standard imaging techniques for the visualization of bony injuries. ${ }^{6}$ In patients presenting with a clinic-radiologic mismatch, MRI has been shown to be a powerful means of identifying soft tissue injuries of the extra neural and intraneural spinal structures. ${ }^{8}$ As the name implies, SCIWORA is an entity in which there are clinical signs of cervical cord damage in the absence of radiologic evidence of vertebral fracture or dislocation. The most common mechanisms of injury are road traffic accidents, followed by sports injuries and fallsthere is an excess of road traffic accidents in the youngest and sportsrelated injuries in adolescents. The mechanism for such injuries in adults was initially postulated to be either hyper flexion-dislocation with immediate reduction by muscular action or temporary prolapse of the cervical disk after forcible flexion.

The ligamenta flava of the cervical spine bulges forward into the canal during hyperextension, producing a marked narrowing of the sagittal canal diameter and cord injury. Additionally, shortening and therefore thickening of the cord during hyperextension as the spinal column shortens may further exacerbate the crowding of intraspinal contents during hyperextension. It is postulated that during hyperextension sprain, the anterior longitudinal ligament can rupture and permit retrolisthesis of the upper segment, leading to cord compression. Elastic recoil of the paraspinous muscles results in spontaneous reduction of the displacement, giving a normal appearance on radiology.

In pediatric patients, the noted mechanisms of injury included hyperextension, flexion, repetitive flexion-extension and longitudinal distraction. It is thought that children are more susceptible to SCIWORA than adults because of the relatively higher elasticity of spinal ligaments allowing for greater deformation forces on the spinal cord, without fracturing or dislocating the vertebrae. Several anatomic features are described, which predispose children to SCIWORA. First, a large head to body ratio and relatively weak neck muscles predispose the unsupported head to large flexion and extension movements. Second, the ligaments and capsules of the spine are more elastic, 
allowing greater force load without tearing. Third, the vertebral body endplate is fragile and breaks away from the centrum relatively easily. Fourth, the high water content of the annulus and inter vertebral disk allow for longitudinal expansion. Fifth, the horizontally positioned vertebral body facet joints permit greater bony motion. Sixth, until roughly the age of 10years, children lack uncinate processes, allowing greater rotary and lateral spinal column movement. Seventh, in young children and infants the vertebral bodies are wedged anteriorly, allowing vertebral column movement. As children grow, the head becomes smaller and the neck musculature stronger. The articulating joints become vertically oriented and the vertebral bodies ossify, increasing stability and decreasing mobility. ${ }^{9}$

The neuroimaging findings are classified into MRI patterns. ${ }^{1}$ In $43 \%$ of patients, no abnormalities were detectable by MRI (Type I), while $57 \%$ had abnormal scan results (Type II). Of these, $6 \%$ had extra neural abnormalities alone (Type IIa), while 38\% showed isolated intraneural lesions (Type IIb). In 13\% of patients, combined extra neural and intraneural abnormalities were described (Type IIc). this was the case with our patient.

The most severe pattern is a complete cord disruption, often in the upper cervical and thoracic spine in younger children, resulting from extreme flexion. The next pattern is a major cord hemorrhage, defined as more than $50 \%$ hemorrhage of cord on axial MRI. The third pattern is a minor cord hemorrhage, representing less than $50 \%$ of the cord on axial MRI. The fourth pattern is edema alone, as is case for our patient, which can have different MRI findings depending on the timing of the MRI after injury. Finally, there are patients who present with acute clinical neurologic findings consistent with SCIWORA and who have no MRI evidence of injury in the cord. It is postulated that there is an injury below the level of detection of current MRI technology. ${ }^{5,10}$ The timing of MRI has also been shown to be critical, and serial scans may detect dynamic intramedullary and extramedullary signal changes or previously undetected abnormalities. ${ }^{11}$ In this context, the concept of delayed-onset SCIWORA is of particular interest: there are reports that clinical symptoms may become evident within minutes of injury but may take as long as10days to develop. ${ }^{12}$ Delayed clinical onset of the neurological findings is of particular importance to our case.

Children with SCIWORA present with a spectrum of neurologic deficits depending on injury location and severity, including transient paresthesias, motor weakness, sensory loss, combined sensor motor deficits, abnormal reflexes, abnormal rectal tone, incontinence, and paralysis. ${ }^{8,13,14}$ Partial cord syndrome, complete cord syndrome, central cord syndrome, Brown Sequard syndrome, and anterior cord syndrome have also been identified. Our patient presented with monoparesis of an upper extremity, which is not considered to be a common neurological sequalae. The initial American Spinal Injury Association impairment scale (AIS) 1 showed a relatively homogenous distribution of injuries (Grade A, 28\%; Grade B, 17\%; Grade C, 31\%; and Grade D, 25\%). At final follow-up, the outcome analysis revealed complete recovery in almost half of the cases (48\%). Initial AIS Grade D was associated with excellent outcome ( $93 \%$ fully recovered), while $37 \%$ of those with Grade B and 54\% of those with Grade C injuries had good-tofair outcomes. Only $13 \%$ of patients with initial AIS Grade an injury made a complete neurologic recovery, and more than two thirds $(69 \%)$ made no improvement at all. The neurological outcome of our patient is within the expected range, according to the aforementioned results. The use of national databases confirms that SCIWORA remains a rare, but important, pediatric diagnosis 10 . With the increasing use of
MRI in the evaluation of neurologic injury after pediatric trauma, our understanding of the spectrum of injuries represented by SCIWORA has evolved. Most current studies highlight the importance of spinal MRI for children with SCIWORA. A classification system of MRI findings led to excellent comparability with published reports1. Statistical analysis revealed a significant association between the extent of initial neurologic impairment, specific MRI patterns, and subsequent outcome.

\section{Conclusion}

Clinicians must maintain a high index of suspicion for occult injury to the spinal cord in children with neurologic deficits after traumatic injury and proceed with advanced neuroimaging for the purpose of accurate diagnosis and to facilitate prognosis. In this regard, we present a case of SCIWORA with indolent (delayed) clinical presentation, unusual neurological examination findings (upper extremity monoparesis without sensory deficits) and a relatively uneventful clinical course.

\section{Acknowledgements}

None.

\section{Conflict of Interest}

The author declares that there is no conflict of interest.

\section{References}

1. Boese CK, Oppermann J, Siewe J, Eysel P, et al. Spinal cord injury without radiologic abnormality in children: a systematic review and meta analysis. J Trauma Acute Care Surg. 2015;78(4):874-882.

2. Augutis M, Levi R. Pediatric spinal cord injury in Sweden: incidence, etiology and outcome. Spinal Cord. 2003;41(6):328-336.

3. Carroll T, Smith CD, Liu X, Bonaventura B, et al. Spinal cord injuries without radiologic abnormality in children: a systematic review. Spinal Cord. 2015;53(12):842-848.

4. Pang D, Wilberger JE. Spinal cord injury without radiographic abnormalities in children. J Neurosurg. 1982;57(1):114-129.

5. Pang D. Spinal cord injury without radiographic abnormality in children, 2 decades later. Neurosurgery. 2004;55(6):1325-1342.

6. Rozzelle CJ, Aarabi B, Dhall SS, Gelb DE, et al. Spinal cord injury without radiographic abnormality (SCIWORA). Neurosurgery. 2013;72(3):227233.

7. Boese CK, Nerlich M, Klein SM, Wirries A, et al. Early magnetic resonance imaging in spinal cord injury without radiological abnormality in adults: a retrospective study. J Trauma Acute Care Surg. 2013;74(3):845-848.

8. Mahajan P, Jaffe DM, Olsen CS, Leonard JR, et al. Spinal cord injury without radiologic abnormality in children imaged with magnetic resonance imaging. J Trauma Acute Care Surg. 2013;75(5):843-847.

9. Platzer P, Jaindl M, Thalhammer G, Dittrich S, et al. Cervical spine injuries in pediatric patients. J Trauma. 2007; 62(2):389-396.

10. Farrell CA, Hannon M, Lee LK. Pediatric spinal cord injury without radiographic abnormality in the era of advanced imaging. Curr Opin Pediatr. 2017;29(3):286-290.

11. Boese CK, Lechler P. Spinal cord injury without radiologic abnormalities in adults: a systematic review. J Trauma Acute Care Surg. 2013;75(2):320 330 . 
12. Kim SH, Yoon SH, Cho KH, Kim SH. Spinal cord injury without radiological abnormality in an infant with delayed presentation of symptoms after a minor injury. Spine. 2008;33(21):E792-E794.

13. Bauman F, Ernstberger T, Neumann C, et al. Pediatric cervical spine injuries: a rare but challenging entity. J Spinal Disord Tech. 2015; 28(7):E377-E384.
14. Shah LM, Zollinger LV. Congenital craniocervical anomalies pose a vulnerability to spinal cord injury without radiographic abnormality (SCIWORA). Emerg Radiol. 2011;18(4):353-356. 